\title{
Benefits and Limitations of Porous Substrates as Biosensors for Protein Adsorption
}

\author{
Thomas D. Lazzara, ${ }^{+}$Ingo Mey, ${ }^{+}$Claudia Steinem, ${ }^{\dagger}$ and Andreas Janshoff ${ }^{*,+}$ \\ ${ }^{\dagger}$ Institute of Organic and Biomolecular Chemistry, Tammannstrasse 2, 37077 Göttingen, Germany \\ ${ }^{\ddagger}$ Institute of Physical Chemistry, Tammannstrasse 6, 37077 Göttingen, Germany
}

Supporting Information

\begin{abstract}
Porous substrates have gained widespread interest for biosensor applications based on molecular recognition. Thus, there is a great demand to systematically investigate the parameters that limit the transport of molecules toward and within the porous matrix as a function of pore geometry. Finite element simulations (FES) and time-resolved optical waveguide spectroscopy (OWS) experiments were used to systematically study the transport of molecules and their binding on the inner surface of a porous material. OWS allowed us to

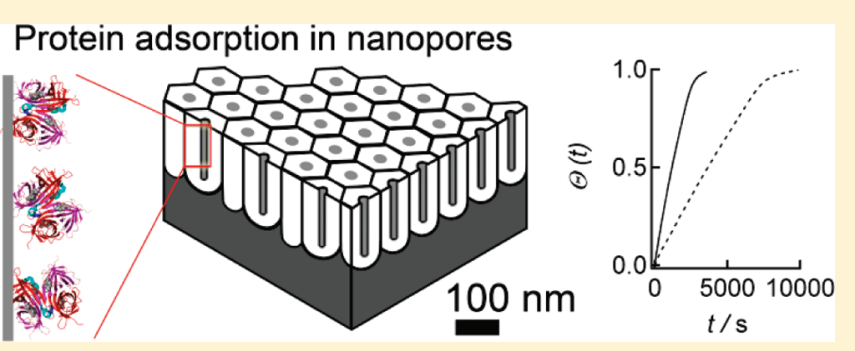
measure the kinetics of protein adsorption within porous anodic aluminum oxide membranes composed of parallel-aligned, cylindrical pores with pore radii of $10-40 \mathrm{~nm}$ and pore depths of $0.8-9.6 \mu \mathrm{m}$. FES showed that protein adsorption on the inner surface of a porous matrix is almost exclusively governed by the flux into the pores. The pore-interior surface nearly acts as a perfect sink for the macromolecules. Neither diffusion within the pores nor adsorption on the surface are rate limiting steps, except for very low rate constants of adsorption. While adsorption on the pore walls is mainly governed by the stationary flux into the pores, desorption from the inner pore walls involves the rate constants of desorption and adsorption, essentially representing the protein-surface interaction potential. FES captured the essential features of the OWS experiments such as the initial linear slopes of the adsorption kinetics, which are inversely proportional to the pore depth and linearly proportional to protein concentration. We show that protein adsorption kinetics allows for an accurate determination of protein concentration, while desorption kinetics could be used to capture the interaction potential of the macromolecules with the pore walls.
\end{abstract}

$\mathrm{B}$ iosensors based on the specific binding of proteins on receptor-functionalized surfaces are among the most widespread analytical tools in biorecognition research. ${ }^{1-5}$ The goal is either to quantify the interaction with the surface in terms of rate constants (adsorption and desorption rate) or association/dissociation constants (adsorption isotherms) to determine the protein concentration in the bulk phase. In either case, knowledge of mass transport limitations is pivotal to obtain reliable data, allowing the determination of biologically relevant interaction parameters. ${ }^{6,7}$ Among the many available experimental techniques to measure rate constants and adsorption isotherms, surface plasmon resonance (SPR) spectroscopy, 8,9 acoustic resonators such as the quartz crystal microbalance, ${ }^{3,10}$ thin-film reflectometry, and optical waveguide methods ${ }^{11,12}$ are the most common ones.

While the adsorption behavior of molecules and macromolecules at planar interfaces is well described ${ }^{6,7,13,14}$ and has been extensively experimentally characterized for practical purposes, ${ }^{15,16}$ the growing number of applications involving mesoporous and macroporous substrates requires a comprehensive investigation of the factors governing macromolecular adsorption within functionalized porous substrates. ${ }^{10,17-22}$ Porous $\mathrm{TiO}_{2}, \mathrm{SiO}_{2}$, or $\mathrm{Al}_{2} \mathrm{O}_{3}$ have recently been investigated as sensor devices to detect protein adsorption on functionalized pore-walls. ${ }^{17,23-28}$ Weiss and co-workers recently reported on a quantitative relationship between pore size, nucleic acid length, and sensor response. ${ }^{29}$ A particularly relevant porous material is anodic aluminum oxide (AAO), which has nonintersecting, hexagonally ordered cylindrical pores that run straight through the film thickness with adjustable pore diameters. $^{30-33} \mathrm{AAO}$ has been used in the selective separation of drug enantiomers $^{34}$ and RNA/DNA oligomers ${ }^{35,36}$ and in the development of on-chip biosensors for protein detection. ${ }^{23}$ Some studies have provided insight into how various parameters, such as ionic strength, ${ }^{37}$ particle size, ${ }^{38,39}$ and porosity ${ }^{11}$ modify the binding efficiency and the transport within porous substrates. However, there is a fundamental need to elucidate which parameters are accessible from a biosensor readout based on porous substrates.

Here, we focused on the protein adsorption kinetics in a porous material using a representative flow cell geometry and solving the convective-diffusion equation with adsorptive boundary conditions employing finite element simulations (FES). FES results were compared with optical waveguide spectroscopy (OWS) experiments on AAO substrates exposed

Received: March 22, 2011

Accepted: June 8, 2011

Published: June 08, 2011 
to avidin solutions. We show that the dominant linear behavior of the time-resolved change in surface concentration can be explained in terms of boundary layer theory, ${ }^{38,40}$ i.e., adsorption kinetics are predominately driven by the flux into the pores. Consequently, the large porous surface area behaves as a perfect sink for protein binding, which results in a depletion of the solution and produces a stationary Lévêque boundary layer. ${ }^{40}$ This makes porous surfaces suitable for potential sensor applications to determine bulk protein concentrations with high accuracy but inadequate to determine the rate constant of adsorption from measuring the surface concentration with time, unless the rate constants are very small.

\section{THEORY}

Mass Balance on a Flat Surface. In this section, we describe the advection-diffusion equation of an adsorbent-carrying liquid with laminar flow perpendicular to an adsorbing surface. For the sake of simplicity, we assume a fully developed flow profile in a one-dimensional rectangular channel (Figure 1A, B). The flow velocity $u_{x}(y)=\gamma y(1-y / b)$ in the $x$-direction is parabolic across the thickness $b$ of the flow cell. $\gamma$ is the wall shear rate $\left(\gamma=2 u_{\max } / b\right)$ and $u_{\max }$ the maximum velocity of the flow in the center of the channel at $y=b / 2$. Ignoring diffusion in the direction of the flow ( $x$-direction), the transient convective diffusion equation governing the adsorption on a flat surface is given as

$$
\frac{\partial c(x, y, t)}{\partial t}+\gamma y\left(1-\frac{y}{b}\right) \frac{\partial c(x, y, t)}{\partial x}=D \frac{\partial^{2} c(x, y, t)}{\partial y^{2}}, \quad 0 \leq y \leq b
$$

with the following initial and boundary conditions:

$$
\begin{array}{r}
\text { at } t=0, \quad c=0 \text { for all } x, y>0 \\
\text { at } x=0, \quad c=c_{\mathrm{b}} \text { for all } y, t \\
\text { at } y=b / 2, \quad c=c_{\mathrm{b}} \\
\text { at } y=0, \quad \frac{\mathrm{d} \Gamma(x, t)}{\mathrm{d} t}=D \frac{\partial c(x, 0, t)}{\partial y} \\
=k_{\mathrm{ad}} c(x, 0, t)\left(\Gamma_{\max }-\Gamma(x, t)\right)-k_{\mathrm{des}} \Gamma(x, t)
\end{array}
$$

and assuming a stationary, fully developed plane Poiseuille flow implying $(\partial c(x, b / 2, t)) /(\partial t)=0 . D$ is the diffusion constant of the protein, $c(x, y, z)$ the concentration of the protein, and $c_{\mathrm{b}}$ the bulk concentration of the protein far away from any surface. $\Gamma(x, t)$ denotes protein surface concentration, $\Gamma_{\max }$ is the maximum surface concentration and $k_{\mathrm{ad}}$ and $k_{\mathrm{des}}$ are the rate constants of adsorption and desorption, respectively. Surface coverage is defined as $\Theta(t)=\Gamma(t) / \Gamma_{\max }$.

Porous Surfaces. The geometry of the flow cell and the porous media used for simulations and experiments is illustrated in Figure 1. Equation 1 describes the mass balance in the flow cell, while absence of convection is assumed inside the pores. At the inner pore walls, adsorptive boundary conditions (eqs $2 \mathrm{a}-2 \mathrm{~d}$ ) are assumed, while reflective boundary conditions are assigned to the pore rims capturing only adsorption within the porous matrix. The boundary condition given in eq $2 \mathrm{~d}$ takes care of the mass balance at the surface assuming first order Langmuir kinetics. The SEM images (Figure 1C, right-hand side) depict

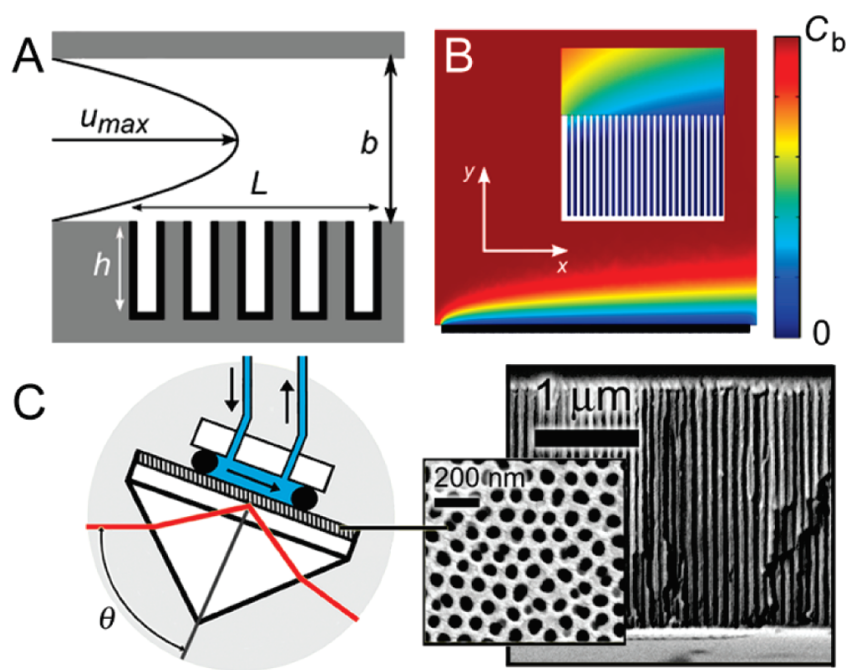

Figure 1. (A) Flow cell geometry used for FES and OWS studies of protein adsorption. For the simulations, equally spaced pores with $R_{\text {pore }}=25 \mathrm{~nm}$ and $h=3.2 \mu \mathrm{m}$ along a distance of $L=120 \mu \mathrm{m}$ were used. The flow chamber has a height of $b=0.5 \mathrm{~mm}$ with a maximum flow velocity of $u_{\max }$. (B) Stationary concentration profile taken after $60 \mathrm{~s}$. (C) Schematic of the OWS setup with SEM images of porous AAO with $R_{\text {pore }}=28 \mathrm{~nm}$.

the top view and cross-section of AAO, showing the ordered cylindrical porous structure of the material. The number of pores per unit area is constant throughout. The membranes have hexagonally packed pores with interpore distances of $p=$ $90-100 \mathrm{~nm}$. The pore radius of the samples can, however, be adjusted between $R_{\text {pore }}=10-40 \mathrm{~nm}$ by isotropic pore widening in phosphoric acid. The pore depth $h$ is controlled by the duration of the anodization.

Simplified Adsorption Kinetics in Porous Media. Assuming that entry into the pores is the rate limiting step of protein adsorption on the pore walls, a stationary boundary layer will be established, as found for perfect sink conditions $\left(c(y=0)=c_{\text {wall }}=0\right)$ on flat surfaces (Supporting Information, Figure S1). The perfect sink assumption for porous media can be rationalized by considering the large number of surface contacts of a protein captured inside a pore of high aspect ratio. Even at low rate constants of adsorption, the sticking probability is strongly enhanced due to the many adhesion trials, i.e. surface collisions.

FES show the validity of boundary layer theory for "perfect sink" conditions with a large parameter space. Only at very low rate constants $\left(k_{\mathrm{ad}}<10^{-3} \mathrm{~m}^{3} \mathrm{~mol}^{-1} \mathrm{~s}^{-1}\right)$, the flux becomes nonstationary and adsorption follows Langmuir kinetics. At the end $(x=L)$ of the adsorption area, the constant flux into the pores can be estimated to be approximately

$$
j=D\left(\frac{\partial c(L, 0)}{\partial y}\right)_{\text {wall }}=\frac{D}{\delta(L)}\left(c_{\mathrm{b}}-c_{\text {wall }}\right)=\frac{D}{\delta(L)} c_{\mathrm{b}}
$$

with the diffusion layer thickness $\delta$ at $x=L$ :

$$
\delta(L)=1.457\left(\frac{D b L}{u_{\max }}\right)^{1 / 3}
$$

The corresponding average diffusion layer thickness is

$$
\langle\delta\rangle=\frac{1}{L} \int_{0}^{L} 1.457\left(\frac{D b x}{u_{\max }}\right)^{1 / 3} \mathrm{~d} x
$$




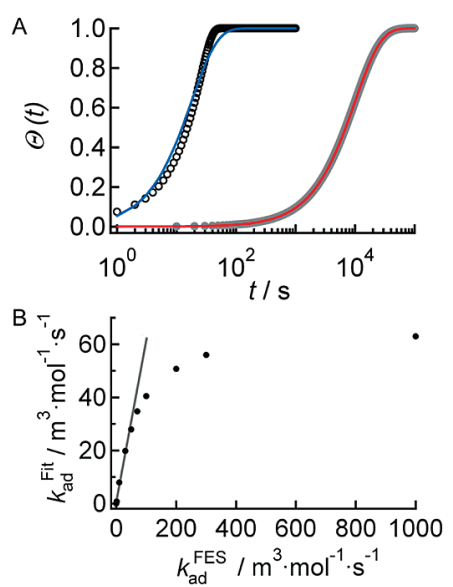

Figure 2. FES of the adsorption process on a flat surface. (A) FES with $k_{\mathrm{ad}}=3 \times 10^{2} \mathrm{~m}^{3} \mathrm{~mol}^{-1} \mathrm{~s}^{-1}(\mathrm{O})$ and $k_{\mathrm{ad}}=10^{-1} \mathrm{~m}^{3} \mathrm{~mol}^{-1} \mathrm{~s}^{-1}(\mathbf{O})$. The solid lines (blue and red) are the results of fitting eq 7 to the simulations providing $k_{\mathrm{ad}}^{\mathrm{Fit}}$. (B) Correlation between $k_{\mathrm{ad}}^{\mathrm{Fit}}$ and $k_{\mathrm{ad}}^{\mathrm{FES}}$; the latter rate constant was used as an input parameter for FES. The solid line represents $100 \%$ correlation.

Consequently, the surface concentration at $x=L$ and the corresponding average surface concentration $\langle\Gamma(t)\rangle$ as a function of time is

$$
\begin{gathered}
\frac{\mathrm{d} \Gamma(L, 0, t)}{\mathrm{d} t}=D\left(\frac{\partial c(L, 0)}{\partial y}\right)_{\text {wall }} \frac{\pi R_{\text {pore }}^{2}}{2 \pi h R_{\text {pore }}+\pi R_{\text {pore }}^{2}} \\
\cong \frac{D}{1.475\left(\frac{D b L}{u_{\max }}\right)^{1 / 3} \frac{R_{\text {pore }}}{2 h} c_{\mathrm{b}}} \\
\Gamma(L, 0, t) \cong 0.68\left(\frac{u_{\max } D^{2}}{d L}\right)^{1 / 3} \frac{R_{\text {pore }}}{2 h} c_{\mathrm{b}} t \\
\langle\Gamma(t)\rangle \cong \frac{D}{\langle\delta\rangle} \frac{R_{\text {pore }}}{2 h} c_{\mathrm{b}} t \approx 0.92\left(\frac{u_{\max } D^{2}}{d L}\right)^{1 / 3} \frac{R_{\text {pore }}}{2 h} c_{\mathrm{b}} t
\end{gathered}
$$

The area ratio between pore entry area $\left(\pi R_{\text {pore }}^{2}\right)$ and overall surface area of a single pore $\left(2 \pi R_{\text {pore }} h+\pi R_{\text {pore }}^{2}\right)$ needs to be taken into account because the flux into the pores (molecules per pore entry area and time) relates to a different area than the rate of adsorption (molecules per wall area and time). This explains why the kinetics are generally slower with increasing surface area. Essentially, eq $6 \mathrm{c}$ predicts that the change in surface concentration for $t \rightarrow 0$ will be linear in bulk protein concentration $c_{\mathrm{b}}$ and inversely proportional to the pore length $h$. Throughout the manuscript we neglect the dependency of the diffusion constant on the pore radius since the protein diameter of avidin is considerably smaller than the pore diameters used in this study (vide infra).

\section{RESULTS AND DISCUSSION}

All FES were carried out using COMSOL within an experimentally accessible parameter space. A standard parameter set was used if not indicated otherwise (range given in brackets): maximum velocity $u_{\max }=10^{-3} \mathrm{~m} \mathrm{~s}^{-1}\left(10^{-5}-10^{-1} \mathrm{~m} \mathrm{~s}^{-1}\right)$, bulk concentration $c_{\mathrm{b}}=10^{-3} \mathrm{~mol} \mathrm{~m}^{-3}\left(10^{-4}-10^{-2} \mathrm{~mol} \mathrm{~m}^{-3}\right), k_{\mathrm{des}}=0, k_{\mathrm{ad}}=$ $10^{3} \mathrm{~m}^{3} \mathrm{~mol}^{-1} \mathrm{~s}^{-1}\left(10^{-4}-10^{4} \mathrm{~m}^{3} \mathrm{~mol}^{-1} \mathrm{~s}^{-1}\right), D=10^{-11} \mathrm{~m}^{2} \mathrm{~s}^{-1}$ $\left(10^{-13}-10^{-8} \mathrm{~m}^{2} \mathrm{~s}^{-1}\right)$, number of pores $=1200$, pore radius $R_{\text {pore }}=25 \mathrm{~nm}$, interpore distance $p=100 \mathrm{~nm}$, length $h=$ $3.2 \mu \mathrm{m}(0.8-9.6 \mu \mathrm{m})$.

Simulating Protein Adsorption Kinetics on Flat Surfaces. We first investigated to what extent the rate constant of adsorption $\left(k_{\mathrm{ad}}\right)$ can be regained from FE-simulations by fitting the integrated form of eq 2 to the FES data, assuming $c(x, 0, t)=c_{\mathrm{b}}$ (Figure 2A):

$$
\frac{\Gamma(t)}{\Gamma_{\max }}=\frac{k_{\mathrm{ad}} c_{\mathrm{b}}}{k_{\mathrm{ad}} c_{\mathrm{b}}+k_{\mathrm{des}}}\left(1-\exp \left\{\frac{t}{\left(k_{\mathrm{ad}} c_{\mathrm{b}}+k_{\mathrm{des}}\right)^{-1}}\right\}\right)
$$

For small $k_{\text {ad }}$ mass transport is not rate limiting, since $c(x, 0, t) \cong$ $c_{\mathrm{b}}$ and kinetics are entirely governed by eq 7 . Albeit adsorption kinetics with larger adsorption rates $\left(k_{\mathrm{ad}}>10 \mathrm{~m}^{3} \mathrm{~mol}^{-1} \mathrm{~s}^{-1}\right) \mathrm{can}$ apparently be modeled with Langmuir adsorption kinetics, the obtained rate constants deviate substantially from the given $k_{\mathrm{ad}}^{\mathrm{FES}}$ values in the simulations. Figure $2 \mathrm{~B}$ shows a correlation between the given $k_{\mathrm{ad}}^{\mathrm{FES}}$ and fitted $k_{\mathrm{ad}}^{\mathrm{Fit}}$ using eq 7 . It becomes obvious that $k_{\mathrm{ad}}^{\mathrm{Fit}}$ is greatly underestimated due to mass transport limitations, which create an almost stationary depletion layer over the surface (Supporting Information, Figure S1).

Simulating Protein Adsorption Kinetics in Porous Media. One might assume that the one-dimensional diffusion of proteins within pores that display a high aspect ratio $\left(R_{\text {pore }} / h<10^{-3}\right)$, might be the rate limiting step of adsorption (for $h \leq 10 \mu \mathrm{m}$ ). In fact, pore dimensions become only relevant on much larger length scales with $h \gg 10 \mu \mathrm{m}$ than considered in this study. Assuming that diffusion is restricted to the $y$-direction, which is justified by the small radii of the pores $\left(R_{\text {pore }}<50 \mathrm{~nm}\right)$, a protein would cover the distance of $1 \mu \mathrm{m}$ in less than $0.1 \mathrm{~s}$ according to $\tau=\left\langle y^{2}\right\rangle / D$ with $\left\langle y^{2}\right\rangle$ being the mean square displacement. More sophisticated numerical analysis confirms that this hands-on argument holds for the pores used in this study (Supporting Information, Figure S2). The impact of pore radius on the diffusion constant of the protein has been neglected since hindered diffusion only becomes relevant if the pore radius is very close to the hydrodynamic radius of the protein. ${ }^{41}$

Generally, we found that the presence of pores slows down the adsorption kinetics with increasing number of pores as compared to a flat surface with the same set of parameters (Figure $3 \mathrm{~A}$ ). Importantly, the adsorption kinetics $(\Theta(t))$ becomes almost linear over a long time period with increasing number of pores associated with a stationary flow into the pores due to a diffusion boundary layer produced by the adsorption capacity of the pores (Figure 1B ). An infinite number of pores essentially represents a perfect sink for proteins approaching the surface which was discussed in the previous section. In order to keep numeric calculations at a manageable level, we used 1200 pores in a $2 \mathrm{D}$ geometry (Supporting Information, Figure S3) throughout the study, which produce a stationary boundary layer similar to that of a reactive wall (Supporting Information, Figures S4 and S5).

For very low adsorption rate constants $\left(k_{\mathrm{ad}}<10^{-3} \mathrm{~m}^{3} \mathrm{~mol}^{-1}\right.$ $\left.\mathrm{s}^{-1}\right)$, eq 7 can safely be used to fit the simulated data reproducing the rate constants $\left(k_{\mathrm{ad}}^{\mathrm{FES}}\right)$ used for the simulation (Figure 3B). For larger $k_{\mathrm{ad}}$, the adsorption is mass transport controlled, which results in a constant flux into the pores and a stationary concentration profile above the pores (Supporting Information, Figure S5).

Figure 3B demonstrates to what extent the adsorption kinetics in porous media deviates from rate limitation (eq 7). The linear initial slope $S_{\mathrm{i}}=\left.(\mathrm{d} \Theta / \mathrm{d} t)\right|_{t \rightarrow 0}$ obtained from FES is plotted as a 

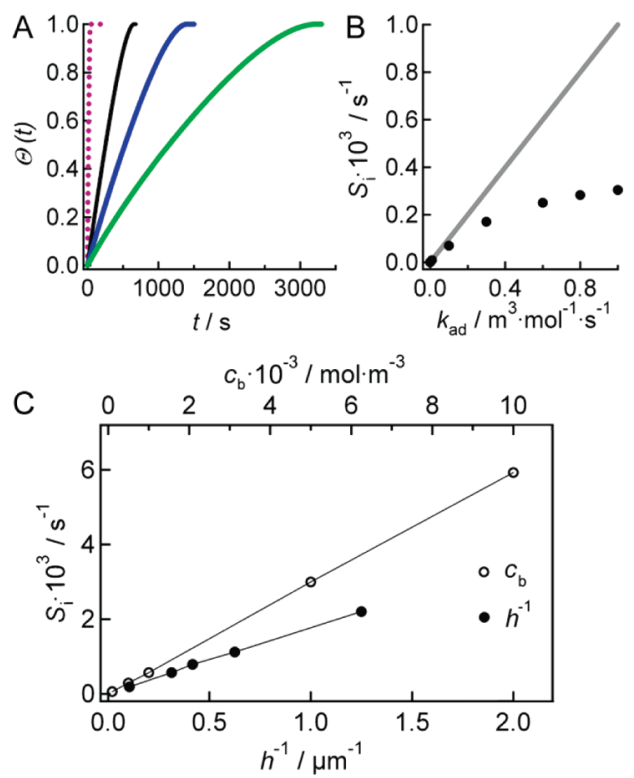

Figure 3. (A) Surface coverage as a function of time for different pore lengths, $h=0.8 \mu \mathrm{m}$ (black), $h=1.6 \mu \mathrm{m}$ (blue), $h=3.2 \mu \mathrm{m}$ (green); the dashed purple line shows the result obtained on a flat surface. (B) Correlation between $k_{\text {ad }}$ used in FES and the resulting initial slope $S_{\mathrm{i}}$. The solid line corresponds to $S_{\mathrm{i}}=k_{\mathrm{ad}} c_{\mathrm{b}}$. (C) Linear dependency of $S_{\mathrm{i}}$ on protein concentration $\left(c_{\mathrm{b}}\right)$ and reciprocal pore depth $\left(h^{-1}\right)$.

function of the adsorption rate constant $k_{\text {ad }}$ used as an input for FES. For very low rate constants, the slope is proportional to $k_{\text {ad }}$ but deviates already for $k_{\mathrm{ad}}>10^{-3} \mathrm{~m}^{3} \mathrm{~mol}^{-1} \mathrm{~s}^{-1}$ leading to a much slower protein adsorption than expected for rate limiting adsorption (Supporting Information, Figure S6). Figure 3C confirms that the initial slope $S_{\mathrm{i}}$ of $\Theta(t)$ essentially follows eqs $6 \mathrm{~b}$ and $6 \mathrm{c}$ and becomes independent of $k_{\mathrm{ad}}$ for $k_{\mathrm{ad}}>1 \mathrm{~m}^{3} \mathrm{~mol}^{-1} \mathrm{~s}^{-1}$. Notably, $k_{\mathrm{ad}}$ is usually in the range of $>100 \mathrm{~m}^{3} \mathrm{~mol}^{-1} \mathrm{~s}^{-1}$ for most molecular recognition events. For instance, low affinity antigen - antibody interactions are reported to exhibit a $k_{\mathrm{ad}}$ in the range of $1 \mathrm{~m}^{3} \mathrm{~mol}^{-1} \mathrm{~s}^{-1}$, while high affinity antigen-antibody or biotin-avidin reactions display rate constants larger than $10000 \mathrm{~m}^{3} \mathrm{~mol}^{-1} \mathrm{~s}^{-1} \cdot 42,43$

In conclusion, replacing the flat adsorbing surface with porous media has one important consequence for the kinetics of protein adsorption on the pore walls. The walls of the pores essentially act as a reacting wall (perfect sink), which produces a stationary concentration profile (depletion layer) that renders adsorption entirely controlled by mass transport into the pores. Porous media are essentially a realization of a perfect sink with respect to boundary layer theory producing the identical concentration profile expected for a flat surface with adsorbing boundary conditions. As a consequence, rate constants of adsorption can only be determined for very low $k_{\text {ad }}$ values $\left(<10^{-3} \mathrm{~m}^{3} \mathrm{~mol}^{-1} \mathrm{~s}^{-1}\right)$.

Adsorption Kinetics of Avidin Binding to Porous AAO. Verification of the theoretical considerations was obtained from protein adsorption experiments on AAO. A rectangular flow cell configuration under laminar flow was used in OWS experiments (Figure 1C). The experimental adsorption surface has about $10^{7}$ pores $/ \mathrm{mm}^{2}$, with cylindrical pores adjusted to a desired pore radius $R_{\text {pore }} \geq 10 \mathrm{~nm}$ (Figure $1 \mathrm{C}$ ). By monitoring the change in the AAO dielectric response, we recorded the electrostatically ${ }^{44}$ driven adsorption kinetics of avidin on the AAO pore walls. With an isoelectric point near $\mathrm{pH} \mathrm{4,} \mathrm{positively} \mathrm{charged} \mathrm{avidin} \mathrm{binds}$ to $\mathrm{AAO}$ at $\mathrm{pH}>5$, while it desorbs at $\mathrm{pH}<3$ (Supporting
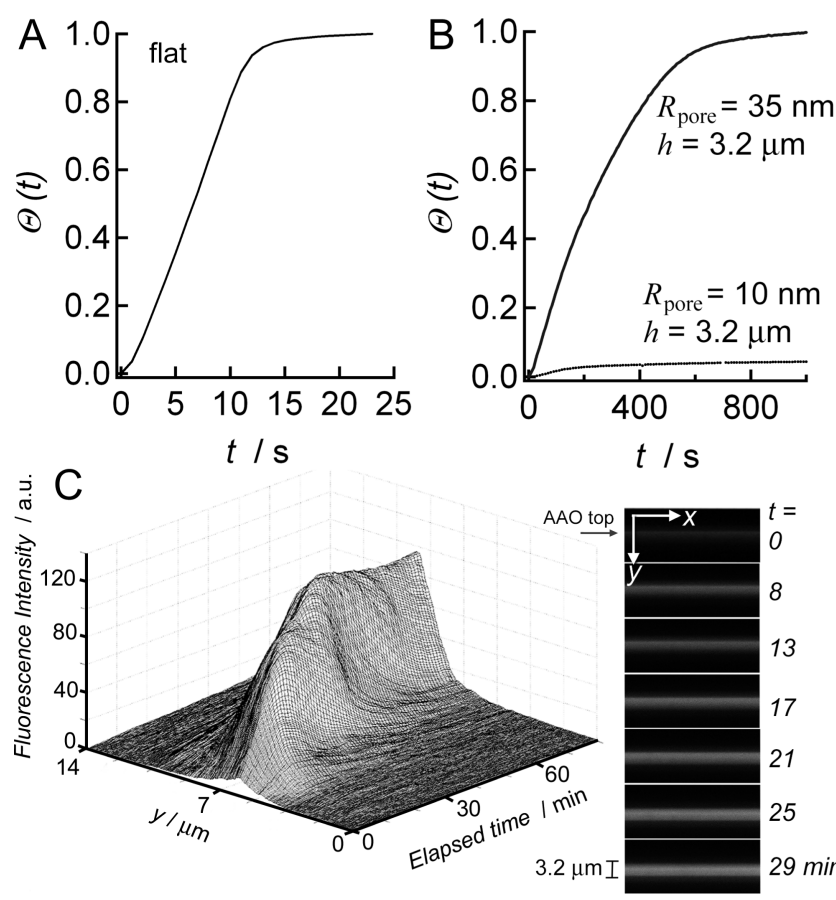

Figure 4. (A) Adsorption kinetics of avidin $\left(c_{b}=1.5 \mu \mathrm{M}\right)$ on a flat negatively charged gold surface measured by SPR. (B) Avidin adsorption kinetics on $\mathrm{AAO}\left(c_{\mathrm{b}}=1.5 \mu \mathrm{M}\right)$ as a function of $R_{\text {pore }}$. Pores with $R_{\text {pore }}=$ $10 \mathrm{~nm}$ are not filled with avidin due to a combined steric and electrostatic hindrance. (C) Left hand side: time evolution of fluorescence, across the $y$-direction, of avidin $\left(c_{\mathrm{b}}=0.45 \mu \mathrm{M}\right)$ adsorbing onto AAO $\left(R_{\text {pore }}=\right.$ $32.5 \mathrm{~nm}, h=3.2 \mu \mathrm{m})$. Right-hand side: vertical slices ( $y$-direction) taken at different times showing the fluorescence increasing across the AAO thickness.

Information, Figure S7). Kinetics were obtained by following the angular shift of a high order TM waveguide mode ${ }^{12}$ (Supporting Information, Figure S8). Generally, we found substantially slower kinetics in comparison to flat surfaces (Figure 4). The adsorption of avidin $\left(c_{\mathrm{b}}=1.5 \mu \mathrm{M}\right)$ under similar conditions on a planar gold surface functionalized with negatively charged mercaptohexadecanoic acid, measured by surface plasmon resonance (SPR), takes about $10 \mathrm{~s}$, which translates into $k_{\mathrm{ad}} \approx 200 \mathrm{~m}^{3}$ $\mathrm{mol}^{-1} \mathrm{~s}^{-1}$, while adsorption within a porous substrate $\left(R_{\text {pore }}=\right.$ $35 \mathrm{~nm}, h=3.2 \mu \mathrm{m}$ ) takes about 60 times longer (Figure 4 ). With the use of $c_{\mathrm{b}}=1.5 \mu \mathrm{M}$ avidin solution, the change in surface concentration with time is approximately linear over a long time period (500-600 s) until the pore wall surfaces start to become saturated with protein and thus the signal slowly levels off over the following 100-200 s. As argued in the previous section, the almost linear regime is predominately governed by the constant flux of protein molecules into the pores due to a stationary diffusion boundary layer that forms as a result of protein depletion. We estimated the avidin flux into an individual pore to be only a few proteins per pore per second.

The increase in refractive index in the saturation regime corresponds to an overall protein layer thickness of $2.8 \pm$ $0.5 \mathrm{~nm}$, averaged over several experiments $(n=20)$ and assuming $\varepsilon_{\text {protein }}=2.1$. This corresponds to about 50\% surface coverage with avidin with dimensions of $4.0 \times 5.5 \times 6.0 \mathrm{~nm}^{3} .{ }^{45}$ We corroborated the OWS adsorption kinetics (Figure 4A,B) with time-resolved confocal laser scanning fluorescence microscopy. This was possible since AAO thin-films are sufficiently transparent with low 

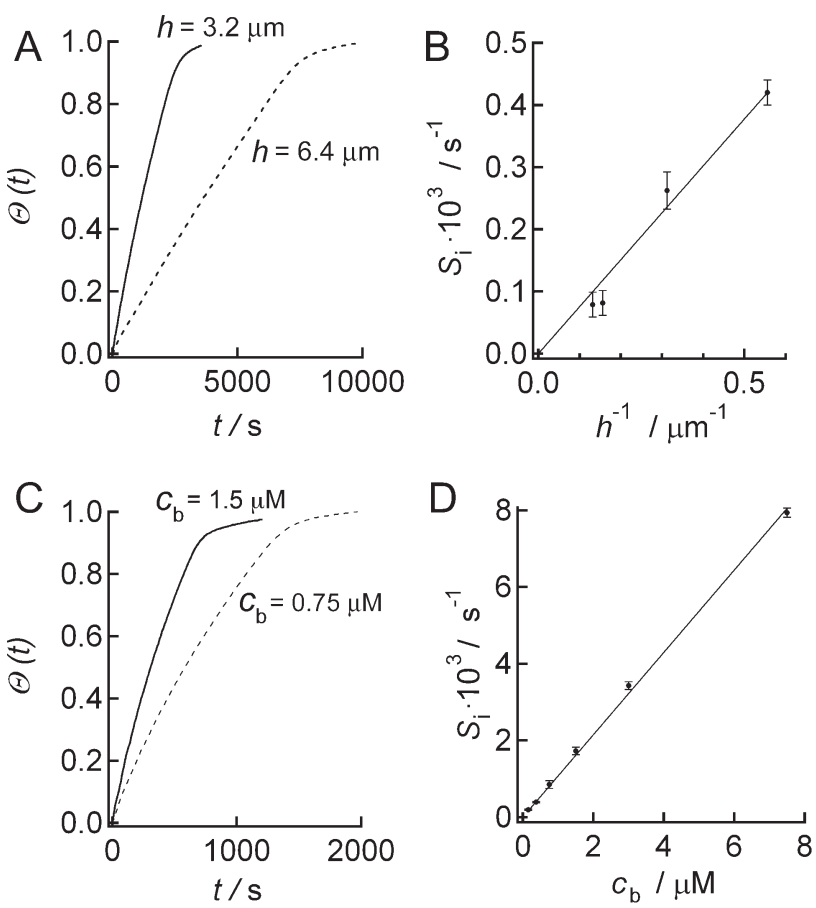

Figure 5. (A) Adsorption kinetics of avidin $\left(c_{\mathrm{b}}=0.45 \mu \mathrm{M}\right)$ on AAO $\left(R_{\text {pore }}=32.5 \mathrm{~nm}\right)$ with two different pore depths. (B) Experimentally determined $S_{\mathrm{i}}$ for avidin adsorption $\left(c_{\mathrm{b}}=0.45 \mu \mathrm{M}\right)$ on AAO $\left(R_{\text {pore }}=\right.$ $32.5 \mathrm{~nm}$ ) as a function of the inverse pore depth $h^{-1}$. (C) Adsorption kinetics of avidin on AAO $\left(R_{\text {pore }}=35 \mathrm{~nm}, h=3.2 \mu \mathrm{m}\right)$ for two different bulk protein concentrations $c_{\mathrm{b}}$. (D) $S_{\mathrm{i}}$ for avidin adsorption on AAO $\left(R_{\text {pore }}=35 \mathrm{~nm}, h=3.2 \mu \mathrm{m}\right)$ measured at different protein concentrations $c_{\mathrm{b}}$.

background fluorescence. We imaged a cross-sectional portion of the AAO by taking sequential slices in the $z$-direction to study the evolution and distribution of the fluorescence signal arising from the adsorption of fluorescently Alexa-488 labeled avidin within AAO $\left(R_{\text {pore }}=32.5 \mathrm{~nm}, h=3.2 \mu \mathrm{m}\right)$. The fluorescence increase ( $y$-direction) with time was essentially linear (Figure 4C), as observed for the adsorption kinetics measured by OWS. By varying the pore radii, we found that the pore radius needed to be adjusted to $R_{\text {pore }}>20 \mathrm{~nm}$, in order to ensure nonhindered pore entry and thus enable pore-filling.

Figure 4B shows that pores with $R_{\text {pore }}=10 \mathrm{~nm}$ are not filled with avidin on our experimental time scale. The early onset of adsorption is attributed to adsorption on the pore rims. Considering that the protein has a hydrodynamic radius of around $3.7 \mathrm{~nm},{ }^{46}$ the local pore radius is substantially reduced to about $5 \mathrm{~nm}$ and electrostatic repulsion and steric hindrance in the poreentrance proximity significantly reduces the entrance probability, i.e. the flux into the pores. Therefore, all following experiments were carried out with $\mathrm{AAO}$ having a pore radii of $R_{\text {pore }}=$ $30-35 \mathrm{~nm}$. In Figure 5, the adsorption kinetics of avidin on AAO substrates as a function of pore depth $h$ and bulk avidin concentration $c_{\mathrm{b}}$ are shown. In good agreement with eq $6 \mathrm{c}$ and Figure 3C, the slope depends linearly on $c_{\mathrm{b}}$ and $h^{-1}$. We obtained $S_{\mathrm{i}}$ by fitting the initial slope of the normalized adsorption kinetics, where the angular shift was converted into coverage $(\Theta(t)=$ $\left.\Gamma(t) / \Gamma_{\max }\right)$.

Desorption of Proteins from Porous Walls. Adsorption kinetics of proteins in porous media is almost exclusively governed by the stationary flux into the pores. It is valid to say that each protein that enters a pore immediately adsorbs on the
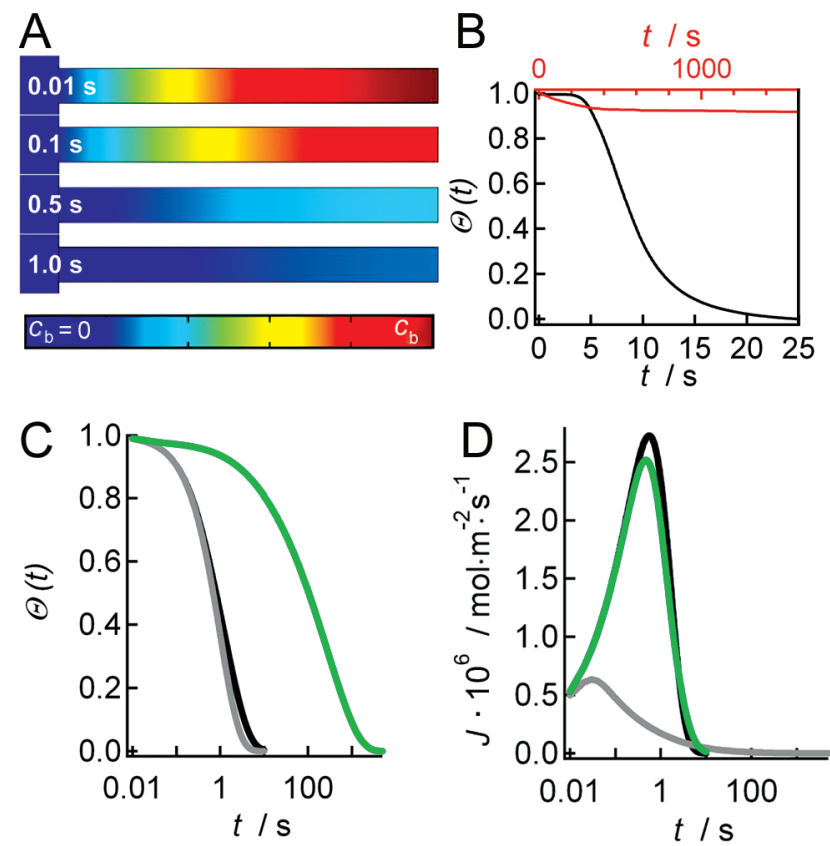

Figure 6. Desorption kinetics of proteins initially covering a single pore. (A) Simulated bulk concentration profile in a single pore at different times. (B) Experimentally measured surface coverage as a function of time for (i) the complete desorption of avidin from the porous surface by reduction of $\mathrm{pH}$ to values smaller than 3 (black curve) and (ii) the incomplete desorption when protein-free buffer is flushed onto the AAO after saturation of the avidin adsorption process (red curve). (C) Simulated desorption kinetics as a function of $k_{\mathrm{ad}}\left(k_{\mathrm{ad}}=10^{3} \mathrm{~m}^{3} \mathrm{~mol}^{-1} \mathrm{~s}-1\right.$ (green), $k_{\mathrm{ad}}=1 \mathrm{~m}^{3} \mathrm{~mol}^{-1} \mathrm{~s}^{-1}$ (gray), $k_{\mathrm{ad}}=10^{-3} \mathrm{~m}^{3} \mathrm{~mol}^{-1} \mathrm{~s}^{-1}$ (black)) with $k_{\mathrm{des}}=$ $1 \mathrm{~s}^{-1}$. (D) Flux $J(t)$ of proteins across $z=0$, the entrance of the pore as a function of $k_{\mathrm{ad}}$ (color coding as in part $\mathrm{C}$ ).

pore wall because of the many surface contacts of the diffusing macromolecule, and thus the increased interaction probability compared to a flat substrate. Until the surface coverage becomes nearly saturated, the rate constants of adsorption and desorption do not influence the adsorption kinetics significantly. A different situation arises if the flow cell is flushed with fresh buffer that is devoid of proteins to measure desorption kinetics. We investigated this situation by simulating the release of proteins from a single pore to demonstrate the essential features of this process (Figure 6). Initially, we assumed that the bulk concentration was zero $\left(c_{\mathrm{b}}=0\right)$, while the coverage of the pore walls was maximal $\left(\Gamma(t=0)=\Gamma_{\max }\right)$. Moreover, we assumed that at the pore entrance, $c(y=0, t)=0$, consistent with fast flushing. The boundary conditions at the pore walls remained $\dot{\Gamma}(t)=k_{\text {ad }} c_{\text {wall }}(t)\left(\Gamma_{\max }-\Gamma(t)\right)-k_{\text {des }} \Gamma(t)$. Figure 6 shows desorption kinetics $(\Gamma(t))$ and also the flux $J$ at $y=0$ from the pores into the flow cell for different $k_{\text {ad }}$. The kinetics are considerably slowed down by an increasing rate of adsorption, which can be attributed to rebinding of the released proteins to free surface sites. This reduces the bulk concentration of proteins in the pores and therefore also reduces the gradient that drives the proteins out of the pore. This is contrary to a flat surface where rebinding of released proteins to the surface is negligible if convection in $x$-direction is fast enough. More surface contacts due to longer pores increase the probability of sticking after desorption and therefore reduce the desorption kinetics accordingly. As opposed to adsorption, the flux out of the pores is time dependent since the concentration gradient 
changes with time and displays a maximum. At short times $(t \rightarrow 0)$, the desorption kinetics is solely driven by $k_{\text {des }}$ with $\Gamma(t)$ $=\Gamma_{\max } \exp \left\{-k_{\text {des }} t\right\}$. The flux increases with time since the bulk concentration in the pore increases due to the growing number of proteins desorbing from the pore walls. However, the decreasing number of molecules leads to a vanishing gradient. Consequently, the flux reaches a maximum that is strongly influenced by the adsorption rate (Figure 6D).

In summary, desorption kinetics bears much richer physics and allows, in principle, one to obtain both $k_{\mathrm{ad}}$ and $k_{\text {des }}$ from flushing the surface with protein-free buffer solution. It requires, however, numeric solutions of the governing mass balance equation due to the absence of a stationary gradient. Experimentally, we observed desorption of avidin from the porous surfaces by lowering the $\mathrm{pH}$ to 2.7 below the isoelectric point of the AAO surface (Figure 6B). While the loading kinetics of a $1.5 \mu \mathrm{M}$ avidin solution on AAO $\left(R_{\text {pore }}=32.5 \mathrm{~nm}, h=3.2 \mu \mathrm{m}\right)$ took $600 \mathrm{~s}$, the release of avidin from the pore-walls devoid of affinity to avidin finished in less than $20 \mathrm{~s}$. Conceivably, sensing schemes that rely on the pore exit in response to external stimuli can be an effective method of utilizing the advantages of a nanoporous substrate for practical applications.

\section{CONCLUDING REMARKS}

The increasing interest in the applications of nanoporous media in biosensor research motivated our studies of the adsorption-desorption kinetics of proteins in porous AAO. Kinetics were investigated as a function of flow properties, pore geometry, and protein concentration both theoretically by means of finite element simulations and experimentally using timeresolved optical waveguide spectroscopy.

We found that adsorption is mainly governed by the rate of protein entrance into the pores giving rise to a linear dependence of surface concentration with time consistent with boundary layer theory. This limits the usage of porous media as transducer or matrixes but bears an overlooked potential. While adsorption rates are out of reach, nanopores can act as a gate and time scales of adsorption can be easily shifted by producing pores of different lengths. Since the surface concentration is strictly proportional to the bulk concentration over very long time, nanoporous matrixes are highly suitable for measuring bulk protein concentrations with high accuracy, almost regardless of the adsorption rates. This can be rationalized by a microscopic picture, in which proteins or any other molecule class with a finite stickiness adsorbs on the surface almost irreversibly due to the large number of surface contacts and the low surface coverage. Essentially, every protein that enters a pore with a high aspect ratio goes through a series of collisions with an almost uncovered surface, until the macromolecule finally adsorbs onto the surface. Desorption leads to subsequent rebinding which in turn increases the dwell time of the protein inside the pores. The high surface area of the porous media and the small entrance area are responsible for this effect.

Flushing the flow cell with protein-free buffer inevitably leads to net desorption and protein release from the porous media. As opposed to the adsorption kinetics, the release from the pores strongly depends on the rate constant of adsorption $\left(k_{\mathrm{ad}}\right)$ since the resident time of the proteins is governed by the rebinding probability. In conclusion, the release kinetics compile both rate of adsorption and rate of desorption. Albeit displaying richer physics, desorption from the pore wall and release out of the pores are essentially more cumbersome to describe in terms of simple equations since fluxes are nonlinear and time dependent.

\section{EXPERIMENTAL SECTION}

AAO Waveguides. AAO thin films (Figure 1C) were fabricated by anodization of $\mathrm{Al}$ foils in $0.3 \mathrm{M}$ oxalic acid, $40 \mathrm{~V}$, at $1{ }^{\circ} \mathrm{C}$. The Al was then removed, $2 \mathrm{~nm}$ of $\mathrm{Cr}$ and $25 \mathrm{~nm}$ of Au were evaporated on the AAO barrier side, and the AAO was finally mounted on LaSFN9 glass slides using an optical adhesive. ${ }^{47}$ AAO thickness was controlled by the anodization time. The pores for all AAO membranes were widened to the desired radius

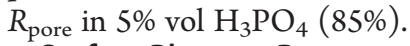

Surface Plasmon Resonance (SPR). SPR measurements were performed on a setup operating at $632.8 \mathrm{~nm}$ in the Kretschmann configuration. ${ }^{48}$

Optical Waveguide Spectroscopy (OWS). OWS measurements of AAO membranes mounted on LaSFN9 glass slides were performed on an SPR setup operating at $\lambda=632.8 \mathrm{~nm}^{37,48,49}$

Fluorescence Microscopy. CLSM measurements were performed on an upright confocal microscope from Carl Zeiss MicroImaging GmbH (LSM 710, Jena, Germany) with a $63 \times$ water immersion objective (WPlan-APO-CHROMAT (NA = 1.0)).

Protein Adsorption Experiments. Avidin was dissolved in $20 \mathrm{mM}$ phosphate buffer, $100 \mathrm{mM} \mathrm{NaCl}, \mathrm{pH}=7$ (PBS) to obtain $1 \mathrm{mg} / \mathrm{mL}$ stock solutions. The flow cell was rinsed with ethanol, followed by PBS. Kinetics were monitored by following the change in a high order waveguide TM-mode. The $1 \mathrm{~mL}$ solution was passed through the flow cell $\left(15 \times 7.5 \times 0.5 \mathrm{~mm}^{3}\right)$ until 1.4 times the dead-volume was washed out, and then the solution was recirculated using a peristaltic pump. The flow rate was kept constant at $0.4 \mathrm{~mL} \mathrm{~min}^{-1}$.

Finite Element Simulations. Calculations were performed with COMSOL Multiphysics 4.1. The models combine a 2D diffusion-convection equation with a 1D Langmuir-type surface adsorption equation. Adsorption occurs only at the inner porewalls. Laminar flow was implemented as a time-constant parabolic velocity field in the flow chamber above the pores.

\section{ASSOCIATED CONTENT}

(5) Supporting Information. Stationary flow profiles and variation of the diffusion layer thickness $\delta$ as a function of $u_{\max }$ and $D$; simulation of $c_{\mathrm{b}}$ as a function of time for a single pore; schematic of the porous system used for FES; adsorption kinetics simulated for 1,300 , and 1200 pores (variation of $S_{\mathrm{i}}$ with the number of simulated pores for low and high $k_{\mathrm{ad}}$ ); flow profiles shown at different times for low and high $k_{\text {ad }}$ (flux as a function of time for a single pore); variation of $S_{\mathrm{i}}$ as a function of $k_{\mathrm{ad}}$; experimental adsorption and desorption curves for avidin on AAO; experimental OWS spectra showing the angular shift obtained for avidin adsorption and adsorption kinetics; and materials and additional experimental details. This material is available free of charge via the Internet at http://pubs.acs.org.

\section{AUTHOR INFORMATION}

\section{Corresponding Author}

*E-mail: ajansho@gwdg.de.

\section{ACKNOWLEDGMENT}

A.J. and C.S. gratefully acknowledge financial support from the DFG (Grants JA 963/8-1 and STE 884/9-1 as well as the Grant SFB 803). T.D.L. acknowledges the award of a doctoral scholarship from the Fonds Québécois de Recherche sur la Nature et les 
Technologies (FQRNT) and additional financial support from the Göttingen Graduate School for Neurosciences and Molecular Biosciences (GGNB).

\section{REFERENCES}

(1) Casero, E.; Vazquez, L.; Parra-Alfambra, A. M.; Lorenzo, E. Analyst 2010, 135, 1878-1903.

(2) Endo, T.; Kerman, K.; Nagatani, N.; Takamura, Y.; Tamiya, E. Anal. Chem. 2005, 77, 6976-6984.

(3) Janshoff, A.; Galla, H. J.; Steinem, C. Angew. Chem., Int. Ed. 2000, 39, 4004-4032.

(4) McPhillips, J.; Murphy, A.; Jonsson, M. P.; Hendren, W. R.; Atkinson, R.; Hook, F.; Zayats, A. V.; Pollard, R. J. ACS Nano 2010, 4, 2210-2216.

(5) Shi, H. Q.; Tsai, W. B.; Garrison, M. D.; Ferrari, S.; Ratner, B. D. Nature 1999, 398, 593-597.

(6) Schuck, P. Annu. Rev. Biophys. Biomol. Struct. 1997, 26, 541-566.

(7) Schuck, P.; Minton, A. P. Anal. Biochem. 1996, 240, 262-272.

(8) Myszka, D. G. Curr. Opin. Biotechnol. 1997, 8, 50-57.

(9) Rich, R. L.; Myszka, D. G. Curr. Opin. Biotechnol. 2000, 11, 54-61.

(10) Janshoff, A.; Steinem, C. Methods Mol. Biol. 2005, 47-63.

(11) Huang, C. J.; Dostalek, J.; Knoll, W. Biosens. Bioelectron. 2010, 26, 1425-1431.

(12) Lau, K. H. A.; Tan, L. S.; Tamada, K.; Sander, M. S.; Knoll, W. J. Phys. Chem. B 2004, 108, 10812-10818.

(13) Rabe, M.; Verdes, D.; Seeger, S. Adv. Colloid Interface Sci. 2011, 162, 87-106.

(14) Schuck, P. Curr. Opin. Biotechnol. 1997, 8, 498-502.

(15) Myszka, D. G.; He, X.; Dembo, M.; Morton, T. A.; Goldstein, B. Biophys. J. 1998, 75, 583-594.

(16) Myszka, D. G.; Morton, T. A.; Doyle, M. L.; Chaiken, I. M. Biophys. J. 1997, 64, 127-137.

(17) Bonanno, L. M.; Kwong, T. C.; DeLouise, L. A. Anal. Chem. 2010, 82, 9711-9718.

(18) Carrasquilla, C.; Li, Y.; Brennan, J. D. Anal. Chem. 2011, 83, 957-965.

(19) DeLouise, L. A.; Miller, B. L. Anal. Chem. 2004, 76, 6915-6920.

(20) Feng, L.; Musto, C. J.; Kemling, J. W.; Lim, S. H.; Zhong, W.; Suslick, K. S. Anal. Chem. 2010, 82, 9433-9440.

(21) Qiao, Y. H.; Wang, D.; Buriak, J. M. Nano Lett. 2007, 7, 464-469.

(22) Janshoff, A.; Steinem, C. Sens. Update 2001, 9, 313-354.

(23) Alvarez, S. D.; Li, C. P.; Chiang, C. E.; Schuller, I. K.; Sailor, M. J. ACS Nano 2009, 3, 3301-3307.

(24) Dancil, K. P. S.; Greiner, D. P.; Sailor, M. J. J. Am. Chem. Soc. 1999, 121, 7925-7930.

(25) Mun, K. S.; Alvarez, S. D.; Choi, W. Y.; Sailor, M. J. ACS Nano 2010, 4, 2070-2076.

(26) Schwartz, M. P.; Alvarez, S. D.; Sailor, M. J. Anal. Chem. 2007, $79,327-334$.

(27) Trivinho-Strixino, F.; Guerreiro, H. A.; Gomes, C. S.; Pereira, E. C.; Guimaraes, F. E. G. Appl. Phys. Lett. 2010, 97.

(28) Walt, D. R. ACS Nano 2009, 3, 2876-2880.

(29) Lawrie, J. L.; Jiao, Y.; Weiss, S. M. IEEE Trans. Nanotechnol. 2010, 9, 596-602.

(30) Li, A. P.; Müller, F.; Birner, A.; Nielsch, K.; Gösele, U. J. Appl. Phys. 1998, 84, 6023-6026.

(31) Li, F. Y.; Zhang, L.; Metzger, R. M. Chem. Mater. 1998, $10,2470-2480$.

(32) Nielsch, K.; Choi, J.; Schwirn, K.; Wehrspohn, R. B.; Gösele, U. Nano Lett. 2002, 2, 677-680.

(33) O'Sullivan, J. P.; Wood, G. C. Proc. R. Soc. London A 1970, $317,511-543$.

(34) Lee, S. B.; Mitchell, D. T.; Trofin, L.; Nevanen, T. K.; Soderlund, H.; Martin, C. R. Science 2002, 296, 2198-2200.

(35) Vlassiouk, I.; Krasnoslobodtsev, A.; Smirnov, S.; Germann, M. Langmuir 2004, 20, 9913-9915.
(36) Gopinath, S. C. B.; Awazu, K.; Fujimaki, M.; Sugimoto, K.; Ohki, Y.; Komatsubara, T.; Tominaga, J.; Gupta, K. C.; Kumar, P. K. R. Anal. Chem. 2008, 80, 6602-6609.

(37) Lazzara, T. D.; Lau, K. H. A.; Abou-Kandil, A. I.; Caminade, A. M.; Majoral, J. P.; Knoll, W. ACS Nano 2010, 4, 3909-3920.

(38) Bird, R. B.; Stewart, W. E.; Lightfoot, E. N. Transport Phenomena; 2nd ed.; John Wiley \& Sons: New York, 2002.

(39) Deen, W. M.; Bohrer, M. P.; Epstein, N. B. AlChE J. 1981, 27, 952-959.

(40) Probstein, R. F. Physicochemical Hydrodynamics: An Introduction, 2nd ed.; John Wiley and Sons: New York, 1994.

(41) Deen, W. M. AlChE J. 1987, 33, 1409-1425.

(42) Rispens, T.; te Velthuis, H.; Hemker, P.; Speijer, H.; Hermens, W.; Aarden, L. J. Immunol. Methods 2011, 365, 50-57.

(43) Stamm, C.; Lukosz, W. Sens. Actuators, B 1994, 18, 183-187.

(44) Wolny, P. M.; Spatz, J. P.; Richter, R. P. Langmuir 2009, 26, 1029-1034.

(45) Livnah, O.; Bayer, E. A.; Wilchek, M.; Sussman, J. L. Proc. Natl. Acad. Sci. U.S.A. 1993, 90, 5076-5080.

(46) Wu, S. C.; Wong, S. L. J. Biol. Chem. 2005, 280, 23225-23231.

(47) Lazzara, T. D.; Lau, K. H. A.; Knoll, W. J. Nanosci. Nanotechnol. 2010, 10, 4293-4299.

(48) Knoll, W. Annu. Rev. Phys. Chem. 1998, 49, 569-638.

(49) Gitsas, A.; Yameen, B.; Lazzara, T. D.; Steinhart, M.; Duran, H.; Knoll, W. Nano Lett. 2010, 10, 2173-2177. 